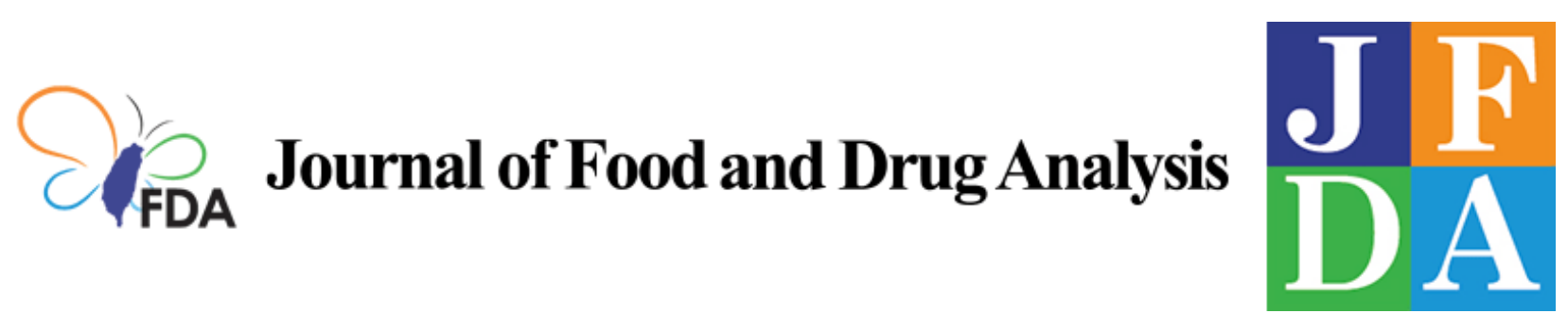

Volume 29 | Issue 4

Article 11

2021

\title{
Quantitative analysis of fragrance allergens in various matrixes of cosmetics by liquid-liquid extraction and GC-MS
}

Follow this and additional works at: https://www.jfda-online.com/journal

Part of the Food Science Commons, Medicinal Chemistry and Pharmaceutics Commons, Pharmacology Commons, and the Toxicology Commons

(c) (i) (9)

This work is licensed under a Creative Commons Attribution-Noncommercial-No Derivative Works 4.0 License.

\section{Recommended Citation}

Lu, Chia-Hui; Fang, Ming-Chih; Chen, Yu-Zi; Huang, Shou-Chieh; and Wang, Der-Yuan (2021) "Quantitative analysis of fragrance allergens in various matrixes of cosmetics by liquid-liquid extraction and GC-MS," Journal of Food and Drug Analysis: Vol. 29 : Iss. 4 , Article 11.

Available at: https://doi.org/10.38212/2224-6614.3373

This Original Article is brought to you for free and open access by Journal of Food and Drug Analysis. It has been accepted for inclusion in Journal of Food and Drug Analysis by an authorized editor of Journal of Food and Drug Analysis. 


\title{
Quantitative analysis of fragrance allergens in various matrixes of cosmetics by liquid-liquid extraction and GC-MS
}

\author{
Chia-Hui Lu ${ }^{a}{ }^{a}$, Ming-Chih Fang ${ }^{b}$, Yu-Zi Chen ${ }^{a}$, Shou-Chieh Huang ${ }^{a}$, Der-Yuan Wang ${ }^{a}$ \\ ${ }^{a}$ Food and Drug Administration, Ministry of Health and Welfare, Executive Yuan, 161-2 Kunyang St., Nangang Dist., Taipei 11561, \\ Taiwan \\ ${ }^{\mathrm{b}}$ Department of Food Science, National Taiwan Ocean University, Keelung, Taiwan
}

\begin{abstract}
Fragrances are the most common chemicals in cosmetics to which people expose every day. However, the unwanted allergic reactions such as contact dermatitis caused by direct contact with fragrances may happen. In Directive 2003/15/EC of the EU, cosmetic product containing one or more of 26 fragrance allergens must be declared on the package label. In addition, commission regulation (EU) 2017/1410 amending Annexes II and III of cosmetic regulation 1223/2009 restricted fragrance chemical of methyl eugenol, and prohibited Lyral, atranol, chloroatranol to be used in cosmetic. In this study, an efficient and sensitive GC-MS method for 3 banned fragrances, 26 fragrance allergens along with restricted methyl eugenol in cosmetics was established. Sample preparation by liquid-liquid extraction was developed by testing various solvent systems to simplify traditional complex extraction methodologies. Validation of the proposed method showed good linearities in a wide concentration ranges of $0.1-10 \mu \mathrm{g} / \mathrm{mL}$. The intra-day and inter-day recoveries were between 84.4 and $119 \%$ with coefficient of variation (CV) below $13.5 \%$. The limit of quantifications (LOQs) of 27 fragrance allergens were in the range of $2-20 \mu \mathrm{g} / \mathrm{g}$. A surveillance study consisted with 82 cosmetics was conducted, among which 31 products claimed fragrance-free. The results showed some fragrance-free claims were false. In the other hand, there were seven cosmetics labeled containing Lyral, but only four were detected. The top fragrance allergens detected in the samples were linalool, limonene, and geraniol. The analysis of fragrance allergens in cosmetics indicated that potential contact allergy related to these products should be considered, even though some fragrance allergens were from natural extracts, such as oak moss absolute.
\end{abstract}

Keywords: Allergen, Cosmetics, Fragrances, GC-MS, Liquid-liquid extraction

\section{Introduction}

$\mathrm{F}$ ragrance substances are derived from natural sources or chemical syntheses. They are organic compounds with pleasant smell, which are enormously used in perfumes and perfumed consumer goods such as cosmetics, detergents and other household products for the purpose of masking unpleasant odors from chemical ingredients [1]. Reports have demonstrated that fragrances in cosmetics are the most common allergens in human daily life [2,3], and may cause allergic contact dermatitis, irritant contact dermatitis, photosensitivity dermatitis, urticaria, and asthma $[4,5]$. According to Directive 2003/15/EC of EU all cosmetics shall declare any of 26 fragrance allergens contained within the product if occurrences above $0.01 \%$ in leave-on and rinse-off products. In addition, the regulation (EU) No. 2017/1410 amending Annexes II (prohibited substances) and III (restricted substances) of Cosmetics Regulation 1223/2009 prohibits the use of Lyral, atranol, chloroatranol, and restricts methyl eugenol [6,7]. Methyl eugenol is recognized as a human carcinogen, and may occur

Received 8 April 2021; revised 1 June 2021; accepted 20 August 2021.

Available online 15 December 2021.

* Corresponding author at: Fax: +886 225631764.

E-mail address: hiflycat04@fda.gov.tw (C.-H. Lu). 
in natural herbal extracts [8]. The limitations of methyl eugenol derived from natural sources in cosmetics are described as following: not exceed $0.01 \%$ in fine fragrance, $0.004 \%$ in eau de toilette, $0.002 \%$ in a fragrance cream, $0.0002 \%$ in other leaveon products and in oral hygiene products, and $0.001 \%$ in rinse-off products.

Sample preparation for cosmetic analysis is crucial because complex matrixes such as high fat, emulsifier, and high solvent may seriously interfere in the determination of fragrances. Various approaches based on the different partition techniques such as liquid-liquid [10], liquid-solid [9], or liquid-gas [10] have been established for the extraction and cleaning. This study adapted liguid-liquid extraction method [11] and investigated various extraction solvents. An effective and sensitive method was developed and validated for the simultaneous determination of 23 restricted, 3 banned, and one restricted fragrance allergens in various types of cosmetic matrix. Surveillance consisted with 31 claimed fragrance-free and 51 perfumed cosmetics in various matrixes such as cream, lotion, shampoo, soaps, deodorants, shower gel, and perfumes purchased from commercial markets was analyzed and discussed.

\section{Materials and method}

\subsection{Chemicals and samples}

Reference standards amyl cinnamyl alcohol, benzyl alcohol, benzyl benzoate, and eugenol were purchased from USP (Rockville, MD, USA). Limonene, methyl-2-octynoate, cinnamyl alcohol, citronellol, citral, $\alpha$-isomethyl ionone, anisyl alcohol, hydroxy citronellol, geraniol, farnesol, linalool, Lilial ${ }^{\circledR}$, Lyral ${ }^{\circledR}$, benzyl salicylate, amyl cinnaml, atranol, hexyl cinnamal, and benzyl cinnamate were purchased from Sigma-Aldrich (St. Louis, MO, USA). Isoeugenol was from AccuStandard (New Haven, USA). Cinnamal and coumarin were from Chem Service (West Chester, PA, USA). Chloroatranol was from Carbosynth (Compton, UK). 4,4'-dibromobiphenyl was from Supelco (Bellefonte, PA, USA). 1,4-Dibromobenzene was from Chem Service (West Chester, PA, USA). A total of 82 cosmetic products including leave on and rinse-of products such as cream, lotion, shampoo, soaps, deodorants, shower gel, and perfumes, were collected from various commercial shops in Taiwan. Samples were stored at room temperature until use.

\subsection{Standard solutions preparation}

Stock solution of individual compounds was prepared by dissolving standard compound each $10 \mathrm{mg}$ in $10 \mathrm{~mL}$ methyl tert-butyl ether, and further diluted into $10-100 \mu \mathrm{g} / \mathrm{mL}$ with methyl tert-butyl ether. The calibration solutions were prepared by diluting standard solutions including internal standard solutions in either matrix solution or methyl tert-butyl ether to the final concentrations of $0.1-10 \mu \mathrm{g} / \mathrm{mL}$. Internal standards, 4,4'-dibromobiphenyl and 1,4dibromobenzene based on EN16274 and a GC-MS method by IFRA [11], were prepared at concentration levels of $1 \mu \mathrm{g} / \mathrm{mL}$ each. Two set of standard solutions of each fragrance compounds were utilized as calibration curves.

\subsection{Sample extraction}

Each sample $0.5 \mathrm{~g}$ was weighted into a $50 \mathrm{~mL}$ amber centrifuge tube. Deionized water $5 \mathrm{~mL}$ and $5 \mathrm{~mL}$ methyl tert-butyl ether was added. The tube was mixed by a Hulamixer ${ }^{\circledR}$ sample mixer (Thermo Fisher inc, Waltham, MA, US) for $30 \mathrm{~min}$, and then water was removed by adding of $5 \mathrm{~g}$ anhydrous sodium sulfate before centrifuging for $30 \mathrm{~min}$ at $3000 \times \mathrm{g}$. The supernatant was collected and filtered with a syringe filter. The filtrate $0.5 \mathrm{~mL}$ was added of $10 \mu \mathrm{L}$ internal standard solution and then dilute to $1 \mathrm{~mL}$ with methyl tert-butyl ether prior to analysis.

\subsection{GC-MS separation}

A GC-MS system consisted with a G188A autosampler, 7890A gas chromatograph, and G7080B single quadrupole mass selective detector (Agilent Technologies, Palo Alto, USA) was utilized. Separation was carried out on a vf-5ms capillary column $(30 \mathrm{~m} \times 0.25 \mathrm{~mm}$ i.d., $0.25 \mu \mathrm{m}$ film thickness, Agilent). Helium as carrier gas was set at a constant flow of $1.0 \mathrm{~mL} / \mathrm{min}$. Sample solution $2.0 \mu \mathrm{L}$ was injected in pulsed splitless mode. GC oven was ramped from 60 to $125^{\circ} \mathrm{C}$ at $3^{\circ} \mathrm{C} / \mathrm{min}, 125-230^{\circ} \mathrm{C}$ at $7{ }^{\circ} \mathrm{C} / \mathrm{min}$, and $230-300{ }^{\circ} \mathrm{C}$ at $20^{\circ} \mathrm{C} / \mathrm{min}$ respectively, and with initial and final hold of 2 and $5 \mathrm{~min}$, respectively. Mass spectrometer was operated in selective ion monitor (SIM) mode. Table 1 presented the specific $\mathrm{m} / \mathrm{z}$ of the target fragrance allergens and internal standards.

\subsection{Method validation}

Neat standard calibration curves were obtained by diluting standard solutions with methyl tert- 
Table 1. Purities and selected fragments of fragrance allergens and internal standard compounds (IS).

\begin{tabular}{|c|c|c|c|}
\hline Compounds & Purity $(\%)$ & CAS No. & $\begin{array}{l}{ }^{*} \text { Quantifier } \\
\text { and Qualifiers }\end{array}$ \\
\hline Amylcinnamic aldehyde & 98 & $122-40-7$ & *129, 117, 202 \\
\hline Anise alcohol & 99.5 & $105-13-5$ & $* 138,137,109$ \\
\hline Atranol & 98.2 & $526-37-4$ & $* 151,152,106$ \\
\hline Benzyl alcohol & 100 & $100-51-6$ & $* 79,107,108$ \\
\hline Benzyl benzoate & 100 & $120-51-4$ & *105, 91, 212 \\
\hline Benzyl cinnamate & 98.5 & $103-41-3$ & *131, 192, 193 \\
\hline Benzyl salicylate & 99.1 & $118-58-1$ & $* 91,92,228$ \\
\hline Chloroatranol & 98.9 & $57074-21-2$ & $* 185,186,187$ \\
\hline Cinnamic alcohol & 98.7 & $104-54-1$ & $* 92,134,115,105$ \\
\hline Cinnamic aldehyde & 97.6 & $104-55-2$ & $* 131,132,103$ \\
\hline Citral: neral & 98 & $5392-40-5$ & $* 69,109,119$ \\
\hline Citral: geraniol & 98 & $5392-40-5$ & $* 69,94,84$ \\
\hline Citronellol & 99 & $106-22-9$ & $* 69,67,81$ \\
\hline Coumarin & 99.5 & $91-64-5$ & $* 118,146,89$ \\
\hline Eugenol & 100 & $97-53-0$ & *164, 149, 131 \\
\hline Farnesol & 98.3 & $4602-84-0$ & $* 69,81,93$ \\
\hline Geraniol & 99 & $106-24-1$ & $* 69,93,123$ \\
\hline Hexylcinnamic aldehyde & 97.6 & $101-86-0$ & $* 129,145,216$ \\
\hline Hydroxycitronellal & 98 & $107-75-5$ & $* 59,71,43$ \\
\hline Isoeugenol & 99.3 & $97-54-1$ & $* 164,149,131$ \\
\hline Lilial & 97.5 & $80-54-6$ & *189, 147, 204 \\
\hline Limonene & 97 & $5989-27-5$ & $* 68,93,67$ \\
\hline Linalool & 99 & $78-70-6$ & $* 93,121,136$ \\
\hline Lyral 1 & 95 & 31906-04-4 & *105, 136, 163 \\
\hline Lyral 2 & 95 & 31906-04-4 & $* 136,105,192$ \\
\hline Methyl-2-octynoate & 99.9 & $111-12-6$ & $* 95,123,79$ \\
\hline Methyl eugenol & 98 & $93-15-2$ & $* 178,147,163$ \\
\hline$\alpha$-Amylcinnamyl alcohol & 100 & $101-85-9$ & $* 133,115,205,204$ \\
\hline$\alpha$-Isomethyl ionone & 91.8 & $127-51-5$ & $* 95,123,79$ \\
\hline 1,4-Dibromobenzene (IS) & - & $106-37-6$ & $* 236,238,234$ \\
\hline 4,4'-Dibromobiphenyl (IS) & - & $92-86-4$ & $* 312,310,314$ \\
\hline
\end{tabular}

butyl ether to final concentrations ranged between 0.1 and $10 \mu \mathrm{g} / \mathrm{g}$. Matrix-matched standard calibration curves were prepared in body lotion extract to final concentration between 0.1 and $10 \mu \mathrm{g} / \mathrm{g}$ in accordance with the sample preparation procedure described in section 2.3. Body lotion contained complex fats and ingredients with low volatilities such as glycerin, caprylic/carlic tryglyceride, ethylhexyl stearate, cetearyl alcohol would be a suitable represented matrix for this study. Limits of quantitation (LOQs) of 27 fragrance allergens were assessed by adding standard compounds into blank matrixes at concentration ranges between 0.1 and $10.0 \mu \mathrm{g} / \mathrm{g}$. The LOQ was estimated as the lowest concentration of analyte that can be quantified with the suitable precision and accuracy using a criteria of $\mathrm{S} / \mathrm{N}$ ratio over 10 . The intra/inter-day accuracy (recovery in \%) and precision (RSD in \%) were assessed by spiking two concentration levels of analytes in 5 replicates. Matrix effects were estimated by comparing the responding area of the analytes between in neat solvent and in the matrix [12] which was calculated by the following formula:

Matrix effect $(\mathrm{ME})=$ area of (analyte in solvent analyte in matrix) $/$ (analyte in solvent) $\times 100 \%$

\section{Results and discussion}

\subsection{Liquid-liquid extraction}

Preliminary tests of extracting fragrance allergens in spiked blank cosmetic by acetone, methanol, and acetonitrile showed interferences and low recoveries. Further, tests of liquid-liquid extraction (LLE) were applied and the recoveries of the fragrance allergens were determined in test solutions consisting of pre-spiked fragrance allergens in blank cosmetic. After clean up, recoveries were determined by the formula described as following. Recovery $(\%)=$ (peak area of analyte in pre-spiked extract/peak area of analyte in post-spiked extract)* $100 \%$. The results showed methyl tert-butyl ether/ 
water partition offered better extraction results over hexane/water, methanol/hexane and acetonitrile/ hexane (Table S1). The results of extremely nonpolar/polar solvent system such as hexane/ water were not satisfied due to anise alcohol, benzyl alcohol, benzyl salicylate, and hydroxycitronellal were relatively polar compounds. The replacement of water to methanol and acetonitrile (lower

Table 2. Validation parameters of the method.

\begin{tabular}{|c|c|c|c|c|c|c|c|c|}
\hline \multirow[t]{2}{*}{ Compounds } & \multirow{2}{*}{$\begin{array}{l}\text { Linear range } \\
(\mu \mathrm{g} / \mathrm{mL})\end{array}$} & \multirow[t]{2}{*}{$r^{2}$} & \multirow{2}{*}{$\begin{array}{l}\text { LOQ } \\
(\mu \mathrm{g} / \mathrm{g})\end{array}$} & \multirow{2}{*}{$\begin{array}{l}\text { Spiked level } \\
(\mu \mathrm{g} / \mathrm{g})\end{array}$} & \multicolumn{2}{|l|}{ Recovery } & \multicolumn{2}{|l|}{$\mathrm{CV}$} \\
\hline & & & & & $\begin{array}{l}\text { Intra-day } \\
(\%)\end{array}$ & $\begin{array}{l}\text { Inter-day } \\
(\%)\end{array}$ & $\begin{array}{l}\text { Intra-day } \\
(\%)\end{array}$ & $\begin{array}{l}\text { Inter-day } \\
(\%)\end{array}$ \\
\hline \multirow[t]{2}{*}{ Amylcinnamic aldehyde } & $0.1-1$ & 0.998 & 2 & 2 & 99.7 & 93.7 & 1.9 & 7.3 \\
\hline & & & & 4 & 98.1 & 97.0 & 0.4 & 4.3 \\
\hline \multirow[t]{2}{*}{ Anise alcohol } & $0.1-1$ & 0.997 & 2 & 2 & 119 & 116 & 3.9 & 3.4 \\
\hline & & & & 4 & 109 & 103 & 7.3 & 7.7 \\
\hline \multirow[t]{2}{*}{ Atranol } & $0.1-1$ & 0.999 & 2 & 2 & 107 & 107 & 6.8 & 4.2 \\
\hline & & & & 4 & 102 & 107 & 2.3 & 3.9 \\
\hline \multirow[t]{2}{*}{ Benzyl alcohol } & $0.1-1$ & 0.998 & 2 & 2 & 100 & 93.9 & 5.2 & 9.5 \\
\hline & & & & 4 & 101 & 96.9 & 5.0 & 5.8 \\
\hline \multirow[t]{2}{*}{ Benzyl benzoate } & $0.1-1$ & 0.998 & 2 & 2 & 84.5 & 87.2 & 0.7 & 4.4 \\
\hline & & & & 4 & 95.6 & 96.1 & 1.2 & 2.9 \\
\hline \multirow[t]{2}{*}{ Benzyl cinnamate } & $0.1-1$ & 0.998 & 2 & 2 & 85.8 & 85.1 & 6.9 & 9.0 \\
\hline & & & & 4 & 94.6 & 94.3 & 0.3 & 3.1 \\
\hline \multirow[t]{2}{*}{ Benzyl salicylate } & $0.1-1$ & 0.995 & 2 & 2 & 106 & 96.0 & 4.2 & 12 \\
\hline & & & & 4 & 104 & 103 & 8.5 & 6.4 \\
\hline \multirow[t]{2}{*}{ Chloroatranol } & $0.1-1$ & 0.998 & 2 & 2 & 102 & 104 & 5.1 & 4.9 \\
\hline & & & & 4 & 102 & 100 & 2.7 & 3.2 \\
\hline \multirow[t]{2}{*}{ Cinnamic alcohol } & $0.1-1$ & 0.998 & 2 & 2 & 115 & 109 & 3.7 & 5.1 \\
\hline & & & & 4 & 113 & 109 & 4.0 & 5.9 \\
\hline \multirow[t]{2}{*}{ Cinnamic aldehyde } & $0.1-1$ & 0.999 & 2 & 2 & 100 & 96.4 & 0.9 & 7.5 \\
\hline & & & & 4 & 100 & 99.5 & 0.8 & 4.4 \\
\hline Citral & $0.1-1$ & 0.998 & 2 & 2 & 86.1 & 92.3 & 8.1 & 7.2 \\
\hline & & & & 4 & 90.9 & 102 & 5.7 & 8.9 \\
\hline Citronellol & $0.1-1$ & 0.996 & 2 & 2 & 95.1 & 99.5 & 12 & 14 \\
\hline & & & & 4 & 93.8 & 102 & 6.3 & 11 \\
\hline Coumarin & $0.1-1$ & 0.997 & 2 & 2 & 89.8 & 88.1 & 0.7 & 4.1 \\
\hline & & & & 4 & 96.8 & 96.4 & 0.4 & 3.3 \\
\hline Eugenol & $0.1-1$ & 0.998 & 2 & 2 & 105 & 109 & 2.9 & 6.0 \\
\hline & & & & 4 & 104 & 109 & 1.9 & 5.9 \\
\hline Farnesol & $0.5-5$ & 0.995 & 10 & 10 & 103 & 99.2 & 11 & 7.2 \\
\hline & & & & 20 & 95.2 & 98.4 & 5.4 & 11 \\
\hline Geraniol & $1-10$ & 0.995 & 20 & 20 & 114 & 110 & 1.0 & 4.3 \\
\hline & & & & 40 & 90.6 & 92.7 & 4.0 & 3.4 \\
\hline Hexylcinnamic aldehyde & $0.1-1$ & 0.998 & 2 & 2 & 98.9 & 96.7 & 1.5 & 5.2 \\
\hline & & & & 4 & 99.9 & 99.4 & 0.6 & 3.6 \\
\hline Hydroxycitronellal & $0.1-1$ & 0.999 & 2 & 2 & 105 & 97.6 & 2.9 & 12 \\
\hline & & & & 4 & 99.2 & 96.0 & 2.1 & 6.1 \\
\hline Isoeugenol & $0.1-1$ & 0.998 & 2 & 2 & 107 & 105 & 2.4 & 5.1 \\
\hline & & & & 4 & 102 & 102 & 2.1 & 5.5 \\
\hline Lilial & $0.1-1$ & 0.998 & 2 & 2 & 90.1 & 87.1 & 0.8 & 4.9 \\
\hline & & & & 4 & 95.9 & 95.0 & 0.5 & 3.3 \\
\hline Limonene & $0.1-1$ & 0.998 & 2 & 2 & 90.1 & 87.7 & 0.8 & 5.5 \\
\hline & & & & 4 & 92.4 & 95.5 & 4.9 & 5.5 \\
\hline Linalool & $0.1-1$ & 0.999 & 2 & 2 & 97.8 & 93.7 & 0.9 & 7.6 \\
\hline & & & & 4 & 92.6 & 97.5 & 0.9 & 4.1 \\
\hline Lyral & $0.1-1$ & 0.999 & 2 & 2 & 101 & 93.1 & 3.6 & 7.4 \\
\hline & & & & 4 & 93.9 & 92.6 & 1.8 & 7.5 \\
\hline Methyl-2-octynoate & $0.1-1$ & 0.997 & 2 & 2 & 104 & 98.5 & 1.6 & 10 \\
\hline & & & & 4 & 102 & 99.2 & 7.8 & 6.9 \\
\hline Methyl eugneol & $0.1-1$ & 0.998 & 2 & 2 & 96.5 & 94.2 & 0.9 & 5.9 \\
\hline & & & & 4 & 99.8 & 99.3 & 0.6 & 3.5 \\
\hline$\alpha$-Amylcinnamyl alcohol & $0.1-1$ & 0.999 & 2 & 2 & 98.5 & 97.8 & 1.5 & 4.4 \\
\hline & & & & 4 & 98.4 & 98.8 & 2.0 & 3.6 \\
\hline$\alpha$-Isomethyl ionone & $0.1-1$ & 0.998 & 2 & 2 & 99.6 & 96.8 & 1.0 & 5.9 \\
\hline & & & & 4 & 99.9 & 99.1 & 0.7 & 3.8 \\
\hline
\end{tabular}


polarities to water) in the liquid-liquid system did improve the recoveries of polar compounds, but some compounds such as cinnamic alcohol, methyl2-octynoate, limonene, and $\alpha$-isomethyl ionone showed decreased recoveries, due to these compound were immiscible in methanol and acetonitrile. Hence, in the LLE partition system, water was remained and hexane was replaced to methyl tertbutyl ether (relatively higher polarity to hexane). This methyl tert-butyl ether/water system showed excellent recovery rates over hexane methanol, acetonitrile, acetone, water, and their mixtures. The water in the extraction system was further removed by sodium sulfate anhydrous. Chromatogram (Fig. S1) showed there was no significant interfere of 27 analytes in a lotion matrix. LLE offered fast sample preparation and removed most of fats by relatively high polar solvent system. The recoveries of analytes obtained were acceptable. Therefore, LLE would be a suitable methodology for preparing sample for GC/MS in complex cosmetic matrix.

\subsection{Method validation}

Fragrance allergens in total 27 compounds ( 24 of 26 EU fragrances, in which 2 are natural extracts; 2 of 3 banned fragrances, in which one is overlapped with 26 EU fragrances; one restricted fragrance, methyl eugenol) were evaluated at the concentration ranges of $0.1-10.0 \mu \mathrm{g} / \mathrm{mL}(0.1,0.2$, $0.5,0.7,1,2,5,7$, and $10 \mu \mathrm{g} / \mathrm{mL}$ ) with the internal standards at $1 \mu \mathrm{g} / \mathrm{mL}$ in duplicates. Good linearity was achieved at the concentrations of $1-10 \mu \mathrm{g} / \mathrm{mL}$ for geraniol, $0.5-5 \mu \mathrm{g} / \mathrm{mL}$ for farnesol, and $0.1-1 \mu \mathrm{g} / \mathrm{mL}$ for other 25 fragrance allergens in this study. The coefficient of determination $\left(\mathrm{r}^{2}\right)$ were all higher than 0.995. The LOQs determined for farnesol was $10 \mu \mathrm{g} / \mathrm{g}$; for geraniol was $20 \mu \mathrm{g} / \mathrm{g}$; for other 25 fragrance allergens were $2 \mu \mathrm{g} / \mathrm{g}$. In recovery studies, the intra-day accuracies of fragrance allergens were between 84.5 and $119 \%$, while the precision (RSD) located in the range between 0.4 and $12 \%$. The inter-day accuracies were obtained between 85.1 and $116 \%$ with precision between 2.9 and $13 \%$ (see Table 2).

\subsection{Matrix effect in GC analysis}

Matrix effects of analytes were shown in Table 3. Significant signal enhanced or suppressed results were observed in most fragrance allergens in this study, suggesting there were either matrix enhancement or suppression for analytes in GCMS analysis of cosmetics. It could be the fatty matrix and some polar ingredients such as glycerin coeluted with analytes. Therefore, matrix-matched calibration curves were suggested for the analysis of various types cosmetics.

Table 3. Matrix effects of fragrances in cosmetics by GCMS analysis.

\begin{tabular}{|c|c|c|c|}
\hline Compounds & $\begin{array}{l}\text { Equation of the } \\
\text { solvent-only } \\
\text { calibration curve }\end{array}$ & $\begin{array}{l}\text { Equation of the } \\
\text { matrix-matched } \\
\text { calibration curves }\end{array}$ & Matrix effect $(\%)$ \\
\hline Amylcinnamic aldehyde & $y=0.7802 x+0.0201$ & $y=0.5789 x+0.0149$ & -25.8 \\
\hline Anise alcohol & $y=0.6286 x-0.0608$ & $y=0.8491 x-0.1057$ & 35.1 \\
\hline Atranol & $y=0.1994 x-0.0367$ & $y=0.5844 x-0.0261$ & 193.0 \\
\hline Benzyl alcohol & $y=0.6550 x-0.0151$ & $y=0.7803 x-0.0112$ & 19.1 \\
\hline Benzyl benzoate & $y=1.5441 x-0.0517$ & $\mathrm{y}=1.2172 \mathrm{x}+0.0570$ & -21.2 \\
\hline Benzyl cinnamate & $y=0.3606 x-0.0660$ & $y=0.5876 x+0.0232$ & 63.0 \\
\hline Benzyl salicylate & $y=1.9756 x-0.3642$ & $y=0.8054 x+0.0796$ & -59.2 \\
\hline Chloroatranol & $y=0.1714 x-0.0333$ & $y=0.3927 x-0.0073$ & 129.0 \\
\hline Cinnamic alcohol & $y=0.0606 x-0.0111$ & $y=0.1591 x-0.0320$ & 162.5 \\
\hline Cinnamic aldehyde & $y=0.9004 x-0.0497$ & $y=1.0459 x-0.0068$ & 16.2 \\
\hline Citral & $y=0.0012 x-0.0454$ & $y=0.0015 x+0.0055$ & 25.0 \\
\hline Citronellol & $y=0.6078 x-0.0003$ & $y=0.1701 x-0.0332$ & -72.0 \\
\hline Coumarin & $y=0.7988 x+0.0474$ & $y=0.5863 x+0.0280$ & -26.6 \\
\hline Eugenol & $y=0.6557 x-0.0373$ & $y=0.5913 x-0.0144$ & -9.8 \\
\hline Farnesol & $y=0.7961 x-0.0599$ & $y=0.3247 x-0.1443$ & -59.2 \\
\hline Geraniol & $y=2.0415 x-0.1315$ & $y=1.9945 x-0.0355$ & -2.3 \\
\hline Hexylcinnamic aldehyde & $y=0.6486 x-0.3410$ & $y=0.4884 x-0.0005$ & -24.7 \\
\hline Hydroxycitronellal & $y=0.7330 x-0.0490$ & $y=0.8613 x-0.0440$ & 17.4 \\
\hline Isoeugenol & $y=0.1946 x-0.0257$ & $y=0.2172 x-0.0121$ & 11.6 \\
\hline Lilial & $y=0.6405 x+0.0089$ & $y=0.4649 x+0.0192$ & -27.4 \\
\hline Limonene & $y=0.65815 x+0.0219$ & $y=0.6985 x+0.0107$ & 6.1 \\
\hline Linalool & $y=0.52964 x-0.0115$ & $y=0.4322 x+0.0003$ & -18.4 \\
\hline Lyral & $y=0.0130 x-0.0007$ & $y=0.0113 x-0.0055$ & -13.0 \\
\hline Methyl-2-octynoate & $y=0.2520 x-0.0156$ & $y=0.3088 x-0.0038$ & 22.5 \\
\hline Methyl eugenol & $y=1.2359 x+0.0923$ & $\mathrm{y}=0.8935 \mathrm{x}+0.0341$ & -27.7 \\
\hline$\alpha$-Amylcinnamyl alcohol & $y=0.4150 x-0.0602$ & $y=0.5491 x-0.0226$ & 32.3 \\
\hline$\alpha$-Isomethyl ionone & $y=0.9301 x+0.0455$ & $y=0.6829 x+0.0037$ & -26.6 \\
\hline
\end{tabular}


Table 4. Contents of fragrances in the products.

\begin{tabular}{|c|c|c|c|c|c|c|c|c|c|}
\hline \multirow[t]{2}{*}{ Compounds } & \multicolumn{3}{|c|}{$\begin{array}{l}\text { Leave-on Products } \\
(\mathrm{n}=21 \text {, lotion, cream) }\end{array}$} & \multicolumn{3}{|c|}{$\begin{array}{l}\text { Leave-on Products } \\
(\mathrm{n}=14, \text { perfume, deodorant) }\end{array}$} & \multicolumn{3}{|c|}{$\begin{array}{l}\text { Rinse-off products } \\
(\mathrm{n}=16 \text {, shampoo, shower gel) }\end{array}$} \\
\hline & $\mathrm{N}$ & Conc. $(\mu \mathrm{g} / \mathrm{g})$ & Mean $(\mu \mathrm{g} / \mathrm{g})$ & $\mathrm{N}$ & Conc. $(\mu \mathrm{g} / \mathrm{g})$ & Mean $(\mu \mathrm{g} / \mathrm{g})$ & $\mathrm{N}$ & Conc. $(\mu \mathrm{g} / \mathrm{g})$ & Mean $(\mu \mathrm{g} / \mathrm{g})$ \\
\hline Amylcinnamic aldehyde & 1 & 47 & 47 & 2 & $81-752$ & 416 & 1 & 48 & 48 \\
\hline Anisyl alcohol & 0 & & & 0 & & & 0 & & \\
\hline Atranol & 0 & & & 0 & & & 0 & & \\
\hline Benzyl alcohol & 7 & $4-6044$ & 1976 & 7 & $9-294$ & 128 & 5 & $5-3510$ & 74 \\
\hline Benzyl benzoate & 7 & $17-3594$ & 676 & 7 & $58-4699$ & 856 & 3 & $33-51$ & 40 \\
\hline Benzyl cinnamate & 0 & & & 2 & $53-108$ & 80 & 0 & & \\
\hline Benzyl salicylate & 6 & $9-2390$ & 494 & 6 & 20-13973 & 3346 & 5 & $16-276$ & 135 \\
\hline Chloroatranol & 0 & & & 0 & & & 0 & & \\
\hline Cinnamic alcohol & 1 & 97 & 97 & 4 & $102-1789$ & 775 & 0 & & \\
\hline Cinnamic aldehyde & 0 & & & 1 & 21 & 21 & 0 & & \\
\hline Citral & 6 & $2-301$ & 145 & 11 & 7-196 & 94 & 5 & $3-117$ & 59 \\
\hline Citronellol & 12 & $4-321$ & 70 & 11 & $4-8100$ & 2200 & 6 & $7-34$ & 21 \\
\hline Coumarin & 8 & $14-249$ & 128 & 10 & $14-4535$ & 559 & 5 & $40-246$ & 113 \\
\hline Eugenol & 2 & $15-220$ & 117 & 4 & $40-200$ & 106 & 5 & $9-1017$ & 425 \\
\hline Farnesol & 3 & $-{ }^{\mathrm{a}}$ & $-^{\mathrm{a}}$ & 4 & $-^{\mathrm{a}}$ & $-^{a}$ & 0 & & \\
\hline Geraniol & 13 & $69-445$ & 269 & 12 & $43-3688$ & 914 & 9 & $68-1827$ & 425 \\
\hline Hexylcinnamic aldehyde & 3 & $75-1038$ & 417 & 5 & $75-17868$ & 4664 & 6 & $93-533$ & 237 \\
\hline Hydroxycitronellal & 5 & $24-196$ & 86 & 9 & $83-4040$ & 926 & 7 & $12-498$ & 263 \\
\hline Isoeugenol & 1 & 15 & 15 & 5 & $36-130$ & 79 & 1 & 43 & 43 \\
\hline Lilial $\AA$ & 4 & $45-3927$ & 1048 & 2 & $7650-15305$ & 11477 & 4 & $134-3958$ & 1331 \\
\hline Limonene & 19 & 5-19092 & 1687 & 13 & $84-5603$ & 1748 & 13 & $4-14798$ & 2492 \\
\hline Linalool & 20 & $12-862$ & 252 & 14 & $7-6574$ & 1557 & 13 & $36-1784$ & 436 \\
\hline Lyral ${ }^{\circledR}$ & 2 & $1-2$ & 2 & 1 & 204 & 204 & 1 & 1787 & 1787 \\
\hline Methyl-2-octynoate & 2 & $2-3$ & 3 & 0 & & & 0 & & \\
\hline Methyl eugenol & 0 & & & 1 & $--^{a}$ & $--^{a}$ & 0 & & \\
\hline$\alpha$-Amylcinnamyl alcohol & 0 & & & 0 & & & 0 & & \\
\hline$\alpha$-Isomethyl ionone & 4 & $11-116$ & 59 & 9 & $105-4124$ & 1822 & 5 & $41-493$ & 163 \\
\hline
\end{tabular}

${ }^{\text {a }}$ Detected but concentration below LOQ.

\subsection{Cosmetic labeling review and contents of fragrances of 51 non fragrance-free products}

Fifty one cosmetics labeled containing fragrance ingredients were examined including 35 leave-on (21 lotion, cream, 12 perfume and 2 deodorants) and 16 rinse-off products. The most frequently identified fragrances were linalool $(91.4 \%)$, limonene $(85.7 \%)$, geraniol $(80.0 \%)$, citronellol $(71.4 \%)$ and coumarin $(51.4 \%)$ in leave-on products. Limonene $(68.8 \%)$, linalool $(62.5 \%)$, coumarin $(43.8 \%)$, citronellol $(37.5 \%)$, geraniol $(31.3 \%)$ and benzyl salicylate $(31.3 \%)$ were the most frequently identified fragrances in rinse-off products. The labeling check results were similar to a previous study which investigated 283 cosmetic labels in Italy [13].

Contents of the target fragrance allergens in the cosmetics were quantitated by matrix-matched calibration and described in Table 4. Fragrances were present in $74 \%(20 / 27)$ of the lotions and creams, mostly linalool $(95 \%)$, limonene $(90 \%)$, geraniol $(60 \%)$, citronellol $(55 \%)$ and benzyl benzoate $(45 \%)$. Perfums and deodorants showed a rate of $82 \%$ $(22 / 27)$ and most frequently identified were linalool $(100 \%)$, limonene $(86.67 \%)$, geraniol $(80 \%)$, coumarin
$(73.33 \%)$ and citral (73.33\%). Fragrance allergens were detected more often in perfumes and deodorants. The most abundant fragrances was limonene $(19092 \mu \mathrm{g} / \mathrm{g})$ in leave-on products, followed by hexyl cinnamic aldehyde $(17868 \mu \mathrm{g} / \mathrm{g})$ and Lilial $(15303 \mu \mathrm{g} / \mathrm{g})$. According to IFRA standards, hexyl cinnamic aldehyde and Lilial were limited in perfumes as $10.7 \%$ and $1.86 \%$, respectively. Most products were clearly and properly labeled, except one cosmetic and one perfume in which limonene $(51 \mu \mathrm{g} /$ g), benzyl alcohol (6044 $\mu \mathrm{g} / \mathrm{g})$, linalool (34 $\mu \mathrm{g} / \mathrm{g}, 12 \mu \mathrm{g} /$ g) and benzyl benzoate $(59 \mu \mathrm{g} / \mathrm{g})$ were found exceed the $0.001 \%(10 \mu \mathrm{g} / \mathrm{g})$ limitation, and linalool $(211 \mu \mathrm{g} / \mathrm{g})$ and hydroxylcitronellal $(485 \mu \mathrm{g} / \mathrm{g})$ were exceed the $0.01 \%(100 \mu \mathrm{g} / \mathrm{g})$ limitation. Methyl eugenol, a restricted fragrance, was found in one perfume sample, but the concentration was below LOQ.

\subsection{Contents of fragrances in claimed fragrance- free cosmetics}

In 31 fragrance-free commercial cosmetics 5 samples were found fragrances. Limonene $(1500 \mu \mathrm{g} / \mathrm{g})$, linalool $(29 \mu \mathrm{g} / \mathrm{g})$, geraniol $(71 \mu \mathrm{g} / \mathrm{g})$ and citronellol $(28 \mu \mathrm{g} / \mathrm{g})$ were found in a hair conditioner sample. A 
lotion, a toothpaste and a facial cleanser were found contained limonene $(18-1500 \mu \mathrm{g} / \mathrm{g})$ and linalool $(5-78 \mu \mathrm{g} / \mathrm{g})$. A facial moisture product declared fragrance-free, but labeled containing benzyl alcohol as preservative and benzyl alcohol was found as $4600 \mu \mathrm{g} / \mathrm{g}$. All of these products were labeled plant extracts or essential oils as their ingredients.

\subsection{Banned fragrance allergens in perfumed cosmetics}

Lyral, atranol, and chloroatranol are banned fragrance substances, and not allowed to use in cosmetic manufacture since 2019 August 31, and as of August 23, 2021, the ban will expand to the selling of remaining stock. In this study, 7 samples were labeled containing Lyral, but only 4 were detected. The highest concentration was $1787 \mu \mathrm{g} / \mathrm{g}$ in a rinse-off product. According to the previous regulation of IFRA, up to $0.2 \%(2000 \mu \mathrm{g} / \mathrm{g})$ Lyral may be used in leave-on product, but lack of description for rinse-off product [14,15]. Atranol and chloroatranol are present in natural extracts, such as Oak moss absolute [16]. However they were not found in all samples.

\section{Conclusion}

In this study, a simple and effective method based on liquid-liquid extraction followed by gas chromatography-mass spectrometry was developed and validated for 27 restricted and banned fragrance allergens in cosmetics. The validated method was applied in the analysis of 81 cosmetic samples containing 31 fragrance-free and 51 perfumed cosmetics. Study results revealed that most cosmetics contained fragrance allergens were clearly dressed in the labels. In few cases, some undeclared fragrance allergens were detected which impose an additional health risk for the consumers.

\section{Acknowledgements}

This study was supported by the grant MOHW109-FDA-M-315-000742 from Food and Drug Administration, Ministry of Health and Welfare in Taiwan, R.O.C.

\section{Appendix}

\section{Supplementary material}

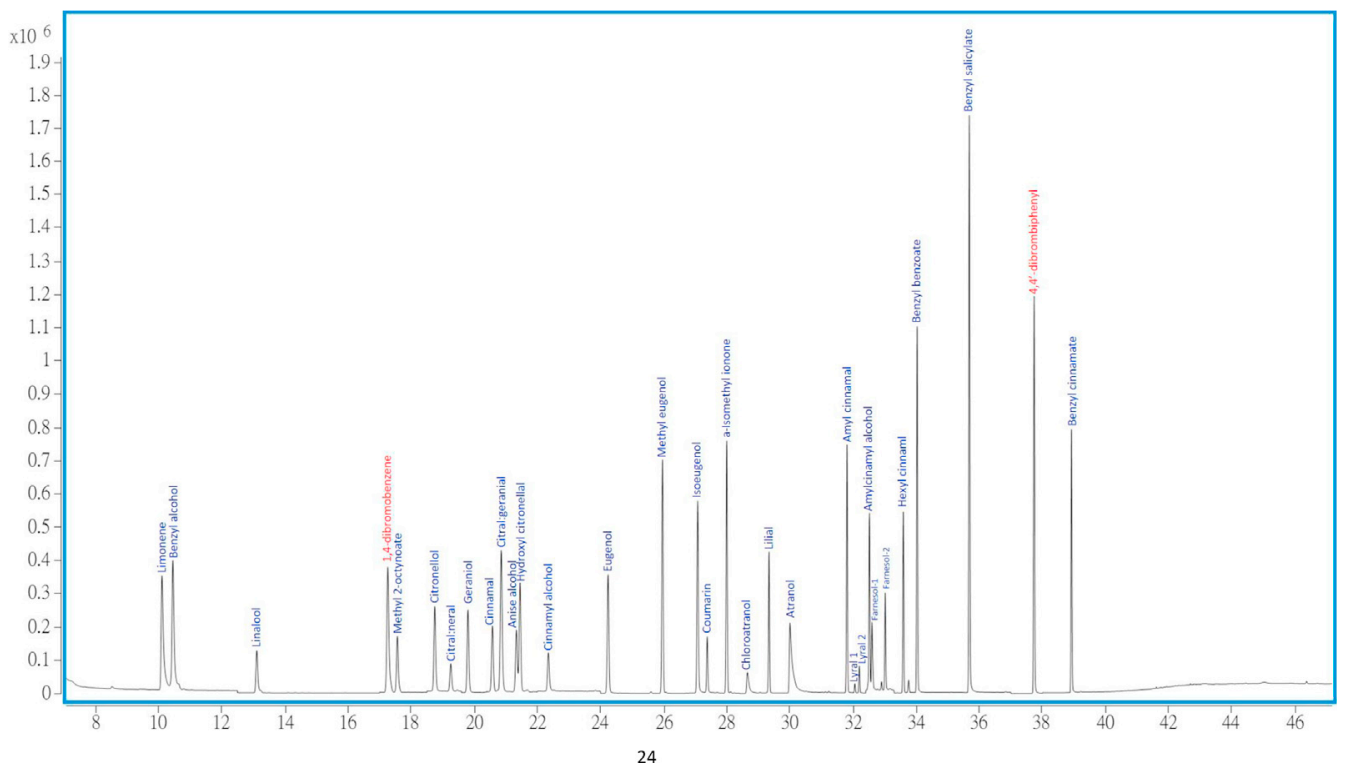

Fig. S1. GC-MS chromatogram (SIM) of a standard mixture of fragrance allergens in lotion matrix. 


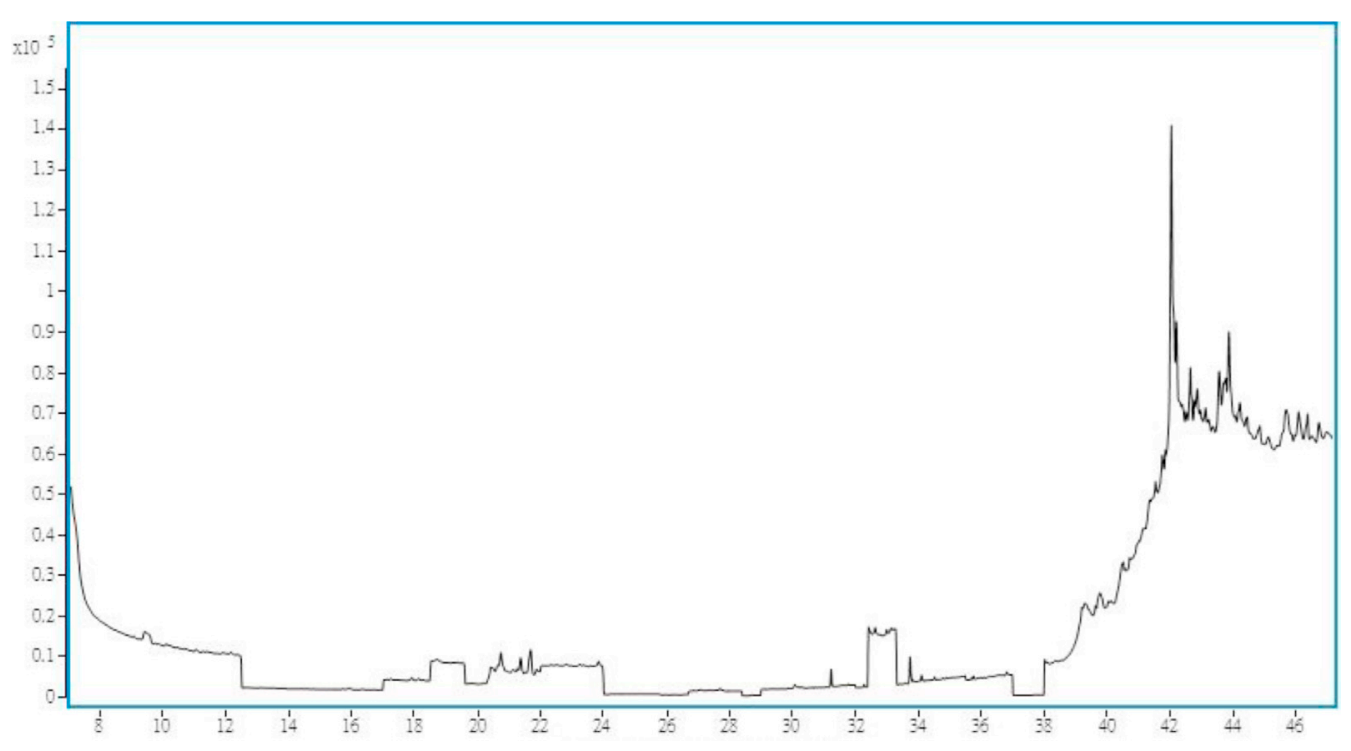

Fig. S2. GC-MS chromatogram (SIM) of the lotion matrix.

Table. S1. Evaluation the extraction efficiency using different organic solvents.

\begin{tabular}{|c|c|c|c|c|}
\hline Compounds & $\begin{array}{l}\text { MTBE/ } \\
\text { water (\%) }\end{array}$ & $\begin{array}{l}\text { hexane/ } \\
\text { water }(\%)\end{array}$ & $\begin{array}{l}\text { methanol/ } \\
\text { hexane }(\%)\end{array}$ & $\begin{array}{l}\text { acetonitrile/ } \\
\text { hexane }(\%)\end{array}$ \\
\hline Amylcinnamic aldehyde & 97.31 & 96.16 & 89.56 & 73.44 \\
\hline Anise alcohol & 86.39 & 12.80 & 78.79 & 77.08 \\
\hline Atranol & 103.44 & 92.98 & 83.30 & 88.73 \\
\hline Benzyl alcohol & 103.45 & 35.82 & 97.44 & 76.58 \\
\hline Benzyl benzoate & 89.14 & 93.09 & 67.00 & 81.76 \\
\hline Benzyl cinnamate & 91.38 & 103.10 & 90.18 & 84.89 \\
\hline Benzyl salicylate & 87.95 & 64.40 & 97.03 & 86.78 \\
\hline Chloroatranol & 85.37 & 92.34 & 65.32 & 76.79 \\
\hline Cinnamic alcohol & 105.07 & 65.93 & 38.05 & 34.54 \\
\hline Cinnamic aldehyde & 96.38 & 89.43 & 90.57 & 81.96 \\
\hline Citral & 89.57 & 94.12 & 67.01 & 77.92 \\
\hline Citronellol & 88.78 & 46.15 & 96.99 & 37.00 \\
\hline Coumarin & 95.45 & 70.87 & 72.38 & 83.06 \\
\hline Eugenol & 98.73 & 78.73 & 65.87 & 83.19 \\
\hline Farnesol & 108.57 & 87.80 & 83.30 & 72.74 \\
\hline Geraniol & 91.74 & 85.46 & 74.80 & 71.44 \\
\hline Hexylcinnamic aldehyde & 93.19 & 95.87 & 73.75 & 91.18 \\
\hline Hydroxycitronellal & 97.00 & 52.31 & 85.75 & 79.83 \\
\hline Isoeugenol & 103.17 & 61.28 & 105.30 & 81.19 \\
\hline Lilial & 98.71 & 93.99 & 76.05 & 72.52 \\
\hline Limonene & 96.05 & 80.66 & 19.28 & 48.94 \\
\hline Linalool & 100.48 & 58.14 & 82.56 & 56.11 \\
\hline Lyral & 98.20 & 97.48 & 85.83 & 70.29 \\
\hline Methyl-2-octynoate & 98.99 & 91.87 & 62.01 & 75.86 \\
\hline Methyl eugenol & 93.43 & 91.44 & 66.25 & 81.87 \\
\hline$\alpha$-Amylcinnamyl alcohol & 97.84 & 99.17 & 89.56 & 83.65 \\
\hline$\alpha$-Isomethyl ionone & 91.73 & 96.87 & 62.27 & 35.38 \\
\hline
\end{tabular}




\section{References}

[1] Scientific Committee on Consumer Safety. Opinion on Fragrance allergens in cosmetic products. European Union; 2011. https://ec.europa.eu/health/scientific_ committees/ consumer_safety/docs/sccs_o_073.pdf. [Äccessed 5 December 2020].

[2] U.S. Food \& Drug Administration. Fragrances in cosmetics 2020; FDA homepage. https://www.fda.gov/cosmetics/ cosmetic-ingredients/fragrances-cosmetics. [Accessed 30 November 2020].

[3] Shih YH, Sun CC, Tseng YH, Chu CY. Contact dermatitis to topical medicaments: a retrospective study from a medical center in Taiwan. Dermatol Sin 2015;33:181-6.

[4] Uter W, Werfel T, Lepoittevin JP, White IR. Contact allergyemerging allergens and public health impact. Int J Environ Res Publ Health 2020;17(7):2404.

[5] Ministry of Environment and food of Denmark Environmental Protection Agency. Survey of allergenic substances in products targeted children-toys and cosmetic products. Copenhagen, Denmark: Ministry of Environment and Food of Denmark; 2016. p. 148

[6] Commission Regulation (EU) 2021/1099 of 5 July 2021 amending Annexes II and III to Regulation (EC) No 1223/2009 of the European Parliament and of the Council on cosmetic products.

[7] European Commission. Opinion concerning methyleugenol - adopted by the SCCNFP during the 14th plenary meeting of 24 october 2000. https://ec.europa.eu/health/ph_risk/ committees/sccp/docshtml/sccp_out126_en.htm. [Accessed 19 December 2020].

[8] National Toxicology Program, Department of Health and Human Services. Methyl eugenol. Report on carcinogens. Fourteenth Edition; 2016. https://ntp.niehs.nih.gov/ntp/roc/ content/profiles/methyleugenol.pdf. [Accessed 19 December 2020].
[9] Celeiro M, Guerra E, Lamas JP, Lores M, Garcia-Jares C, Llompart M. Development of a multianalyte method based on micro-matrix-solid-phase dispersion for the analysis of fragrance allergens and preservatives in personal care products. J Chromatogr A 2014;1344:1-14.

[10] Desmedt B, Canfyn M, Pypea M, Baudewyns S, Hanot V, Courselle P, et al. HS-GC-MS method for the analysis of fragrance allergens in complex cosmetic matrices. Talanta 2015;131:444-51.

[11] The International Fragrance Association Analytical Working Group. Analytical method to quantify 57 suspected allergens (and isomers) in ready to inject fragrance material by gas chromatography and mass spectrometry. 2016. https:// ifrafragrance.org/docs/defaultsource/guidelines/23754_gd_ 2017_04_11_ifra_analytical_method_to_quantify_57 suspected_allergens_(and_isomers)_in_ready_to_inject fragrance_materials_by_gc-ms-(3).pdf?sfvrsn=ad55ac1_6. [Accessed 10 November 2019].

[12] Zhou W, Yang S, Wang PG. Matrix effects and application of matrix effect factor. Bioanalysis 2017;9(23):1839-44.

[13] Panico A, Serio F, Bagordo F, Grassi T, Idolo A, Giorgi MD, et al. Skin safety and health prevention: an overview of chemicals in cosmetic products. J Prev Med Hyg 2019;60: $50-7$.

[14] International fragrance association. IFRA Standards 43th Amendment; 2009 IFRA standards documentation. https:// ifrafragrance.org/safe-use/standards-documentation. [Accessed 11 October 2020].

[15] Audrain H, Kenward C, Lovell CR, Green C, Ormerod AD, Sansom J, et al. Allergy to oxidized limonene and linalool is frequent in the U.K. Br J Dermatol 2019;171(2):292-7.

[16] Johansen JD, Bernard G, Giménez-Arnau E, Lepoittevin JP, Bruze M, Andersen KE. Comparison of elicitation potential of chloroatranol and atranol-2 allergens in oak moss absolute. Contact Dermatitis 2006;54:192-5. 